\title{
Temperature-Dependent Raman Scattering of ZnSe Nanowires
}

\author{
Lingcong Shi', Chunrui Wang ${ }^{1 *}$, Jiale Wang1, Zebo Fang2*, Huaizhong Xing1 \\ ${ }^{1}$ Department of Applied Physics, State Key Laboratory for Modification of Chemical Fibers and Polymer Materials, Donghua University, \\ Shanghai, China \\ ${ }^{2}$ Department of Physics, Shaoxing University, Shaoxing, China \\ Email: *crwang@dhu.edu.cn, *csfzb@usx.edu.cn
}

How to cite this paper: Shi, L.C., Wang, C.R., Wang, J.L., Fang, Z.B. and Xing, H.Z. (2016) Temperature-Dependent Raman Scattering of $\mathrm{ZnSe}$ Nanowires. Advances in Materials Physics and Chemistry, 6, 305-317. http://dx.doi.org/10.4236/ampc.2016.612029

Received: November 27, 2016

Accepted: December 13, 2016

Published: December 16, 2016

Copyright $\odot 2016$ by authors and Scientific Research Publishing Inc. This work is licensed under the Creative Commons Attribution International License (CC BY 4.0). http://creativecommons.org/licenses/by/4.0/

\begin{abstract}
We present a Raman scattering study of $\mathrm{ZnSe}$ over a temperature range from 80 to $580 \mathrm{~K}$. Both of TO and LO modes of ZnSe nanowires are observed a red-shift with elevating temperature, and its intrinsic broadening is larger than that of bulk counterpart. The opticalphonon lifetime of ZnSe nanowires are longer than that of nanoparticles but shorter than that of bulk, which can be attributed to the different surface to volume ratios. Some overtones and multi-phonon peaks are also observed at high temperature due to the larger wave vector $\boldsymbol{q}$. The $2 \mathrm{TA}_{1}(K)$ and multi-phonon peaks $\mathrm{TA}(X, K)+\mathrm{LA}(X)$ appears at the temperature that is higher than room temperature. The intensity of overtones acoustics $\operatorname{TA}(L)$ elevates with increasing temperature while the surface mode disappears at temperature higher than $296 \mathrm{~K}$. Detailed investigations on temperature-dependent phonon characteristics provide essential component for understanding confined phonons effects at small scale.
\end{abstract}

\section{Keywords}

Thermal Evaporation, ZnSe Nanowires, Raman Scattering, Phonon, Lifetime

\section{Introduction}

ZnSe has attracted intense research interest due to their advanced optical and electrical properties and potential applications in room-temperature continuous-wave laser, lightemitting diodes, blue and green light emitting devices [1] [2] [3]. Recently, one-dimensional nanomaterials have been extensively studied due to its novel and superior properties and potential applications as building blocks in nano-scale electronics [4] [5] [6] [7]. Consequently, different forms of one-dimensional ZnSe nanostructures [6] [8]-[19] have been synthesized in pursuing improved physical properties. Good knowledge of 
the vibration properties of this material is essential to understand transport properties and phonon interaction with the free carriers, both of which have great impact on optoelectronic device performance [10]. In polar semiconductors, carriers already excited to the conduction band relax toward their ground state mainly by Fröhlich interaction with the longitudinal optical phonons [11] [12] [13]. Thus, the dynamics of the phonon population strongly affects the performance of high speed optoelectronic devices. Raman scattering measurements are well suitable to obtain information about the materials quality of wide band gap semiconductors, as well as to analyze some more specific aspects of lattice dynamics, such as isotopic effects, phonon lifetimes [14] [15] [16] [17] [18]. Therefore, extensive investigations on lattice dynamics of various ZnSe nanostructures by using Raman spectroscopy have been reported [19] [20] [21] [22]. However, the majority of Raman studies are carried out only at room temperature, barely at low temperature or merely at high temperature. Few investigations are done at room temperature together with local low and high temperature at the same time. It is well known that variation of temperature has proven to be a powerful probe for understanding electronic and optical phenomena in condensed phases. Changes in temperature can vary inter-atomic distances and vary the overlap among adjacent electronic orbitals [23]. Thus, temperature dependence of phonon modes may provide useful information on the electronic states of energy transfer and electron-phonon coupling [13] [24]. Moreover, some Raman investigations on one-dimensional $\mathrm{ZnSe}$ nanomaterials remain controversial or indistinct [19] [25] [26] [27] [28]. Furthermore, the phonons are related to the properties such as thermal expansion, entropy, specific heat, thermal conductivity, electrical conductivity, optical and dielectric properties, diffusion mechanism, phase change phenomena [29] [30] [31] [32]. So it is important to understand phonon characteristic in one-dimensional $\mathrm{ZnSe}$ nanomaterials changing with temperature.

In this paper, we present systematic investigations on temperature-dependent phonon characteristics of $\mathrm{ZnSe}$ nanowires in order for better understanding the temperature dependent behavior of phonons, which provide essential component for understanding confined phonon effects at small scale.

\section{Experiment}

ZnSe nanowires were synthesized via one-step thermal evaporation as described in our previous work [33], which is easily operated and repeatable. Briefly, ZnSe powders were located at the center of the furnace and a silicon wafer covered with Au was placed downstream away the furnace center to collect the products. The furnace was then pumped down to a base pressure of $\sim 1.2 \times 10^{2} \mathrm{~Pa}$. Argon gas was introduced into the tube at a constant flow rate of $50 \mathrm{sccm}$ (standard-state cubic centimeter per minute). The total pressure was kept at $\sim 2.2 \times 10^{2} \mathrm{~Pa}$ during the experiment process. The furnace was maintained at $1250^{\circ} \mathrm{C}$ for 1.5 hours before it was cooled down to room temperature.

The as-fabricated ZnSe nanowires were characterized by X-Ray Diffraction (XRD), 
field emission scanning electron microscopy (FESEM) and transmission electron microscope (TEM). Raman spectroscopy of the ZnSe nanowires samples under different temperature varying between $80 \mathrm{~K}$ and $580 \mathrm{~K}$ were carried out using Renishaw in ViaReflex micro-Raman spectroscopy system with $532 \mathrm{~nm}$ radiation. The low-temperature Raman spectra were recorded by contacting the sample with the cold finger of a Dewar with liquid nitrogen as the coolant. The high-temperature Raman data were obtained by using a minitype furnace to heat the sample stage. All the Raman spectra were recorded when the set temperature was stable, and all the data were obtained from the same point on the sample. The spectral resolution was less than $1 \mathrm{~cm}^{-1}$ and the focal area of the laser is about $1 \mu \mathrm{m}^{2}$. A series of temperature dependent Raman spectra at different points of our samples are collected and all of them are almost identical to the same one. All the data analyzed in the sections below are treated carefully to reduce the errors.

\section{Results and Discussion}

The X-ray diffraction (XRD) pattern of the as-synthesized ZnSe nanowires is shown in Figure 1(a). All these diffraction peaks can be indexed as the zinc blende phase $\mathrm{ZnSe}$ without other impurities peaks, indicating that the product is a pure compound. The diffraction peaks of $\mathrm{Au}$ and $\mathrm{Si}$ come from the catalyst and the Si substrate. Sharp peaks with small full width at half maximum (FWHM) revel a good crystallinity of our samples. Figure 1(b) presents the SEM image of ZnSe nanowires with good uniformity and wire-like morphology. The nanowires are of $50-150 \mathrm{~nm}$ in diameter with several tens of micrometers in length. Detailed microstructures of the as-grown nanowires were further investigated using transmission electron microscopy (TEM) and selected-area electron diffraction (SAED). Figure 1(c) shows a typical TEM image of ZnSe nanowire. The nanowire shows a uniform diameter of $100 \mathrm{~nm}$ along its whole length. The corresponding selected area electron diffraction (SAED) pattern and the high-resolution TEM (HRTEM) image taken from part of an individual nanowire are shown in Figure 1(c) and display a $\langle 01 \overline{1}\rangle$ zone-axis SAED pattern.

Both From HRTEM image and SAED pattern, we can find that ZnSe nanowire is single-crystal grown along with $<111>$ direction and there is no mismatch or defect in this nanowires.

Figure 2 shows the room-temperature (296 K) Raman spectrum of ZnSe nanowires. Five peaks at $137.5,189.0,203.5,251.0$ and $290.0 \mathrm{~cm}^{-1}$ are observed. The peaks at 203.5 and $251.0 \mathrm{~cm}^{-1}$ are attributed to the scatterings of the transverse optical $(\mathrm{TO}(I))$ and longitudinal optical $(\mathrm{LO}(I))$ phonon modes of $\mathrm{ZnSe}$ [34]. The peak around $290 \mathrm{~cm}^{-1}$ may be originated from the defects in ZnSe nanowires according to the reference [26]. However, since there are no defects observed in $\mathrm{ZnSe}$ nanowireshere, thus we tend to assign this mode to the multi-phonon peak: $\mathrm{TA}(X, K)+\mathrm{LA}(X)$ [19]. The attribution of the peaks at 137.5 and $189.0 \mathrm{~cm}^{-1}$ still remain controversial and indistinct [19] [25] [27] [28] as mentioned in the introduction section. Actually, simply assigning these peaks to

a certain mode are not rigorous with this resolution, because all these modes energy are 


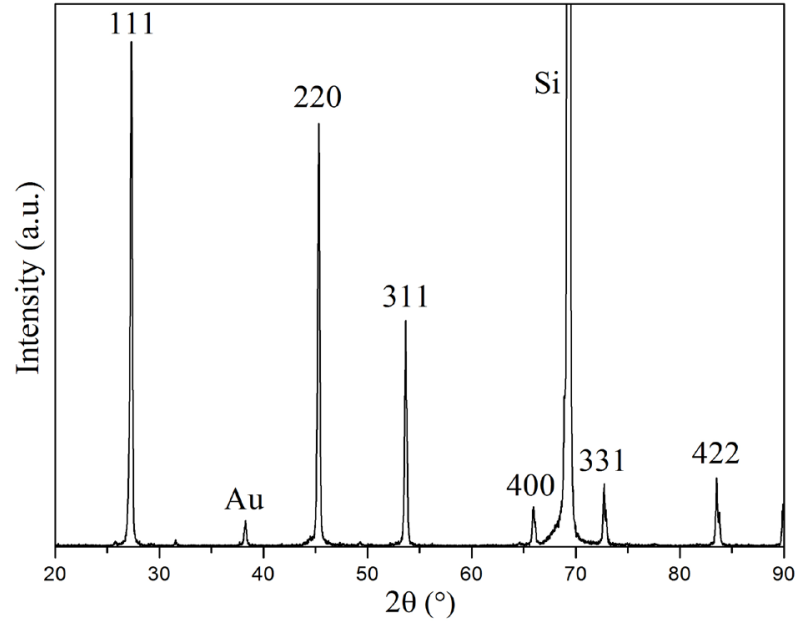

(a)

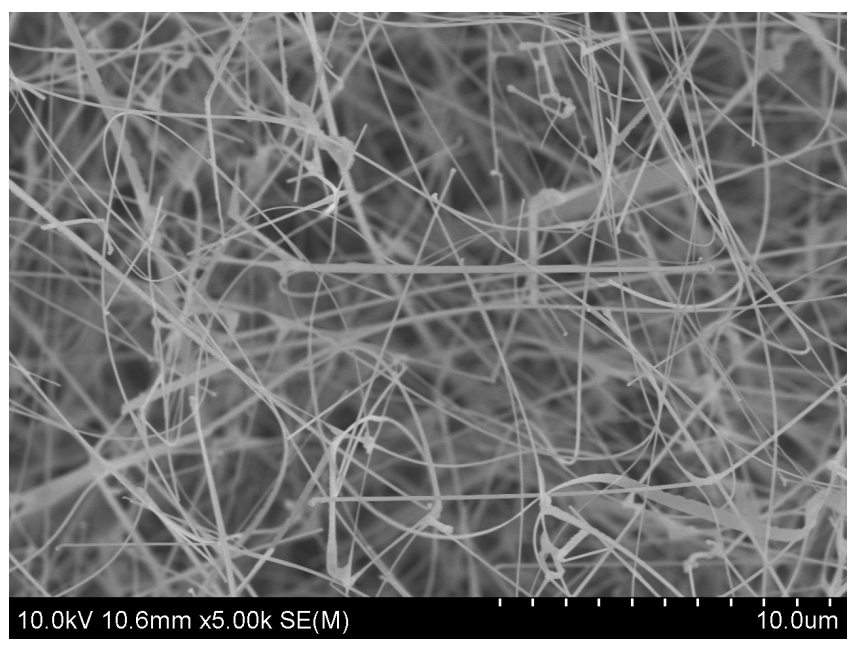

(b)

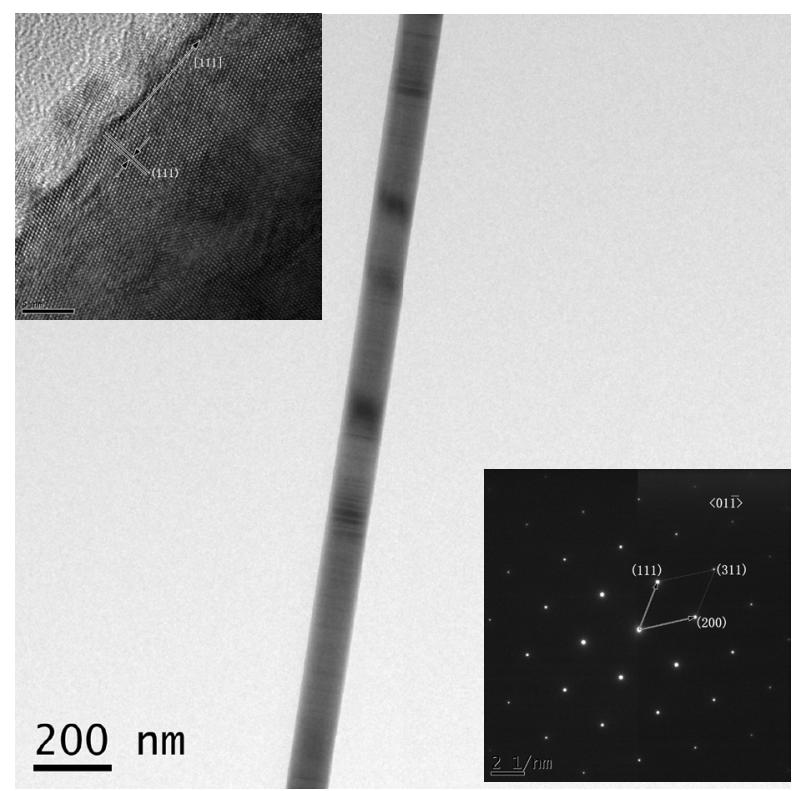

(c)

Figure 1. The (a) XRD pattern, (b) SEM image and (c) TEM image of as-fabricated ZnSe nanowires. The upper and lower insets in (c) are the corresponding HRTEM image and SAED pattern, respectively.

very close and they cannot be distinguished from each other in the phonon spectrum [35]. However, a qualitative relationship can be obtained from the phonon spectrum [35] [36]: $\omega_{\mathrm{TA}}(L)<\omega_{\mathrm{TA}}(X) \approx \omega_{\mathrm{TA} 2}(K)<\omega_{\mathrm{TA} 1}(K)<100 \mathrm{~cm}^{-1}<\mathrm{LA}(X)<200 \mathrm{~cm}^{-1}$. We can tentatively assign the two peaks at 137.5 and $189.0 \mathrm{~cm}^{-1}$ to $2 \mathrm{TA}(L)[28]$ and $2 \mathrm{TA}_{1}(K)$ [19], respectively, on the basis of the qualitative relationship.

To investigate the anharmonicity and understand the electron-phonon interactions in ZnSe nanowires, the temperature-dependent Raman studies of ZnSe nanowires were carried out by varying the temperature starting from nearliquid-nitrogen temperature $(\sim 80 \mathrm{~K})$ until $580 \mathrm{~K}$, which is presented in Figure 3. At all the temperature, there are strong scattering intensities of both TO and LO phonon modes. However, the intense 


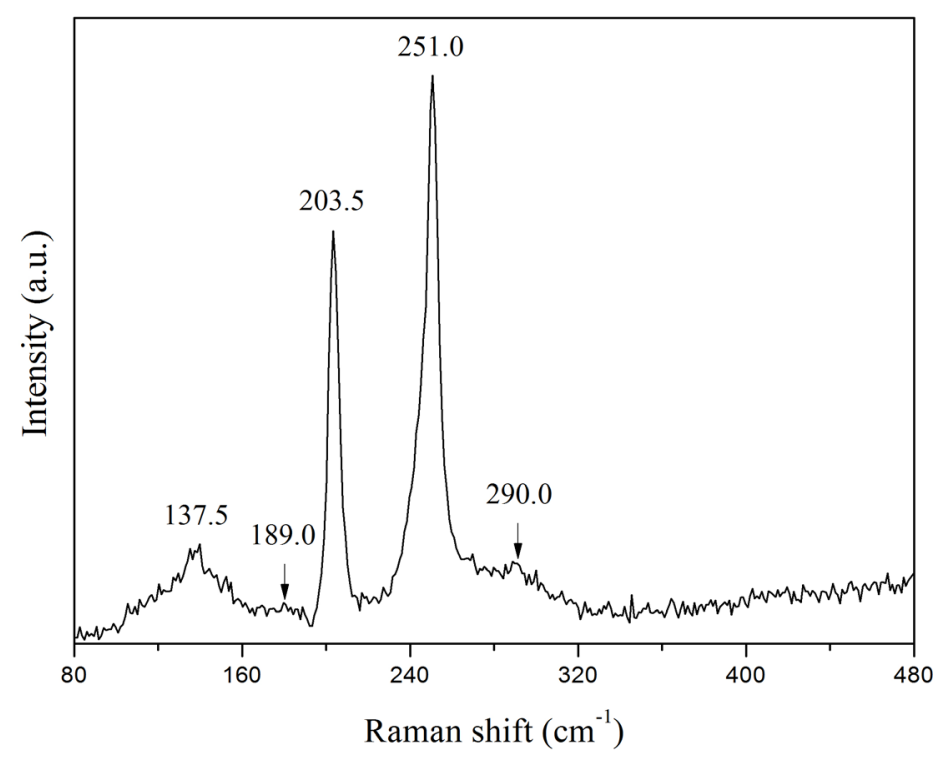

Figure 2. Raman spectra of $\mathrm{ZnSe}$ nanowires measured at room temperature.

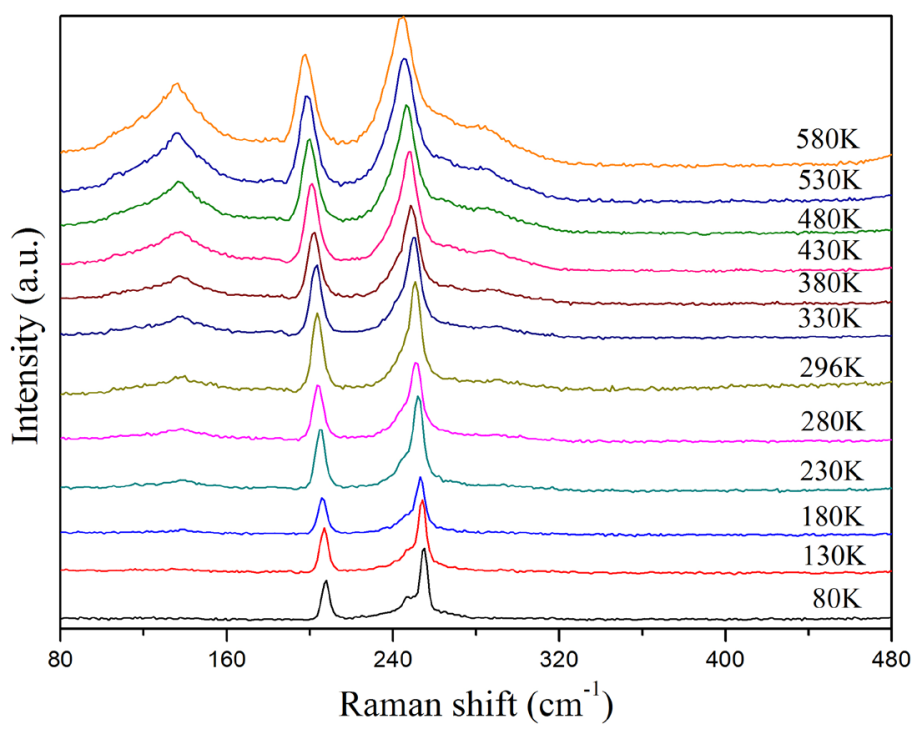

Figure 3. Temperature-dependent Raman Spectra of ZnSe nanowires.

ties of modes associated with acoustics phonons are sensitive to temperature variation. The $2 \mathrm{TA}_{1}(K)$ and multi-phonon peaks $\mathrm{TA}(X, K)+\mathrm{LA}(X)$ appears only above the room temperature. With the temperature changing from $80 \mathrm{~K}$ to $580 \mathrm{~K}$, the intensity of overtones acoustics $\mathrm{TA}(L)$ increases, while the surface mode [37] [38] disappears when arriving at room temperature. A discussion on modes associated with acoustics phonons and surface mode will be discussed latter. Note that, for the TO-phonon and LOphonon modes, there are asymmetry broadenings and redshifts in the Raman line shapes with the increasing temperature, which can be ascribe to the anharmonicity in the vibrational potential. The temperature variations of the linewidths and line centers of the TO- and LO-phonon modes at the Brillouin zones can be given by [39] [40]: 


$$
\begin{aligned}
& \omega(T)=\omega_{0}+C\left\{1+\frac{2}{e^{x}-1}\right\}+D\left\{1+\frac{3}{e^{y}-1}+\frac{3}{\left(e^{y}-1\right)^{2}}\right\} \\
& \Gamma(T)=\Gamma_{0}+\mathrm{A}\left\{1+\frac{2}{e^{x}-1}\right\}+B\left\{1+\frac{3}{e^{y}-1}+\frac{3}{\left(e^{y}-1\right)^{2}}\right\}=\Gamma_{0}+\Gamma_{1}+\Gamma_{2}
\end{aligned}
$$

where $\Gamma_{0}$ is the broadening due to the disorder and boundary scattering in crystal, the second part of the equation is the damping part induced due to the three- and fourphonon process cubic and quartic anharmonicities, respectively. The $\mathrm{x}$ and $\mathrm{y}$ in the above formulae can be written as:

$$
x=\frac{h c \omega_{0}}{2 k_{B} T} \quad y=\frac{h c \omega_{0}}{3 k_{B} T}
$$

where $\omega_{0}$ is the intrinsic frequency of the optical phonon, $A, B, C$ and $D$ are anharmonic constants, $h$ is Plank's constant, $c$ is the speed of light in vacuum, $k_{B}$ is the Boltzmann constant.

Figure 4 shows the temperature-dependent peak positions and linewidths of optical phonon mode of the ZnSe nanowires. Both peak position and linewidth of TO and LO modes have redshifts and broadening. The theoretical curves are in good agreement with the experimental points. The fitted values of constants involved in Equation (1) and (2) for the best theoretical fit to the experimental TO mode phonons are: $\omega_{0}=$ 209.2, $C=-0.013, D=-9.2 \mathrm{E}-7, \Gamma_{0}=3.3, \mathrm{~A}=0.0016, B=4.0 \mathrm{E}-6 \mathrm{~cm}^{-1}$; while refer to LO mode phonons: $\omega_{0}=256.4, C=-0.020, D=1.2 \mathrm{E}-6, \Gamma_{0}=5.9, A=-0.0023, B=$ $2.7 \mathrm{E}-5 \mathrm{~cm}^{-1}$. When comparing the anharmonic term constants $(\mathrm{A}, \mathrm{B}, \mathrm{C}, \mathrm{D})$ between TO and LO mode, the values of the cubic anharmonic constants of both TO and LO modes are much bigger than those of quartic constants. It means that the three-phonon process is dominant and the four-phonon decay process is subordinate [41]. The intrinsic frequency of both $\mathrm{LO}$ and TO modes downshifts and the intrinsic broadenings are larger compared with the bulk $\mathrm{ZnSe}[42]$. Moreover, the constants $|\mathrm{A}|$ and $|\mathrm{C}|$ are decreasing in nanoparticles than in bulk [42]. It indicates that anharmonic effect is more important in bulk than in $\mathrm{ZnSe}$ nanowires.

The linewidth is reciprocally related to the phonon lifetime of the decay process, so we can use a simple relation $\tau=1 / \pi \mathrm{c} \Gamma$ [42] to estimate the lifetime of phonons in terms of uncertainty principle, which is showed in Figure 5. One can find that the lifetimes of both TO and LO modes are decreasing with the increasing temperature. The reason is that the number of phonons is increasing with the elevating temperature. The increasing phonons numbers cut down the scattering mean free path, which raise the chance of scattering between the phonons, and thus reduce the lifetimes. The ratio $\tau_{1} / \tau_{0}$ obtained by calculating the ratio $\Gamma_{0} / \Gamma_{1}$, which varies from 4.1 to 29.6 for LO mode when the temperature decreases from $580 \mathrm{~K}$ to $80 \mathrm{~K}$. It is much larger in $\mathrm{ZnSe}$ nanowires than in bulk [38], this implies that the phonon decay due to anharmonic effects is important for bulk. It is observed that the phonon lifetime in $\mathrm{ZnSe}$ nanowires is 


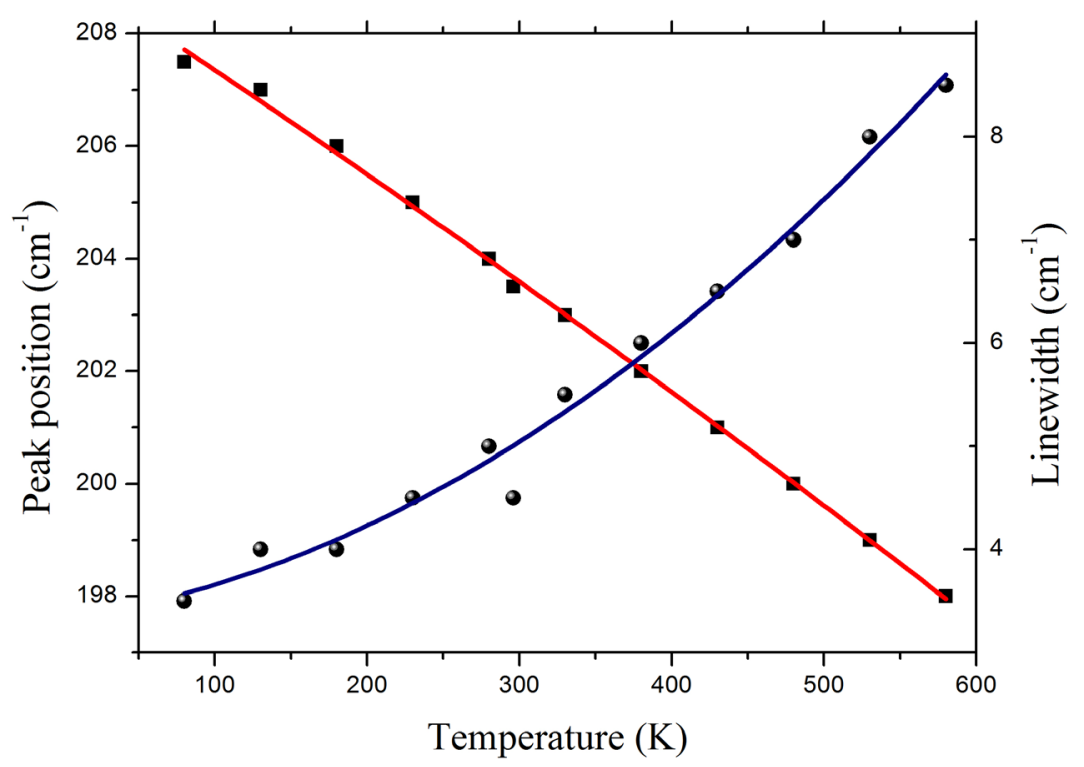

(a)

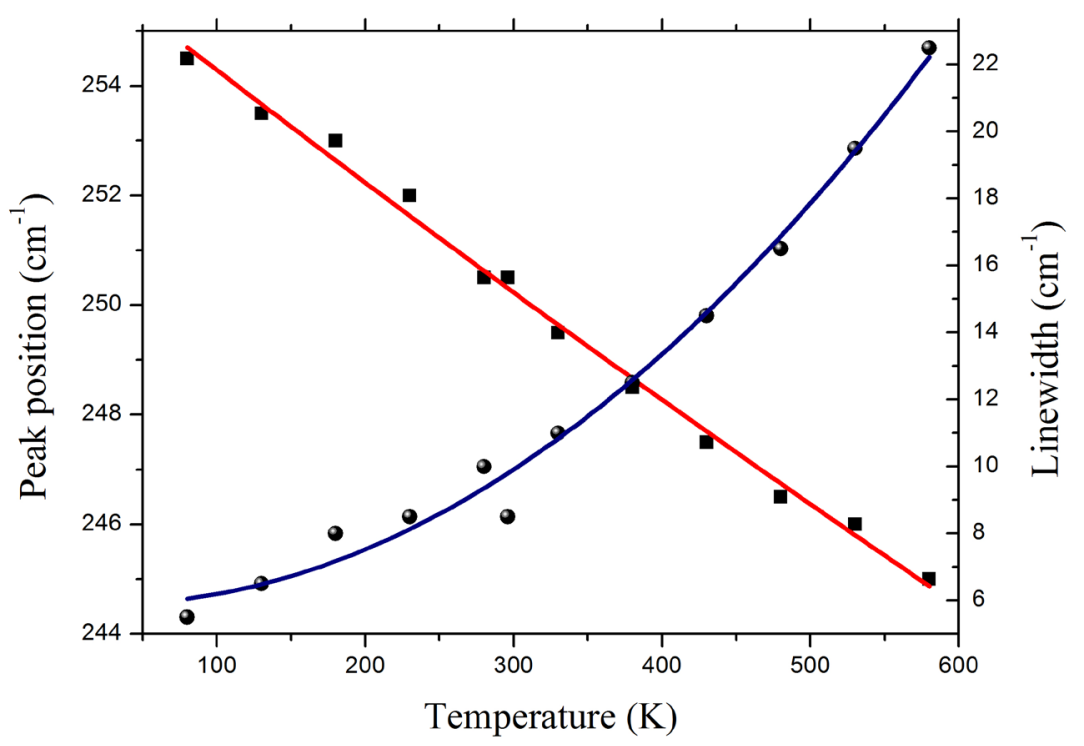

(b)

Figure 4. Temperature-dependent line center and linewidth variation of (a) TO mode and (b) LO mode in the $\mathrm{ZnSe}$ nanowires.

longer than that of ZnSe nanoparticles [38] while shorter than that of the bulkZnSe [42], which can be attributed to the increasing scattering chances with the surface. With the reducing of the dimension, the surface to volume ratio will go up. There are more probabilities to scatter with the surface, which also reduce the phonon lifetimes.

It is noted that the $\operatorname{TA}(L)$ and $\operatorname{TA}(K)$ overtones at 137.5 and $189 \mathrm{~cm}^{-1}$ only appear at a relatively high temperature. Since a higher temperature allows more scattering processes with a large values $\boldsymbol{q}$, the interaction between the acoustic phonons and the energy band electrons becomes more significant [43]. Therefore, more acoustic pho- 


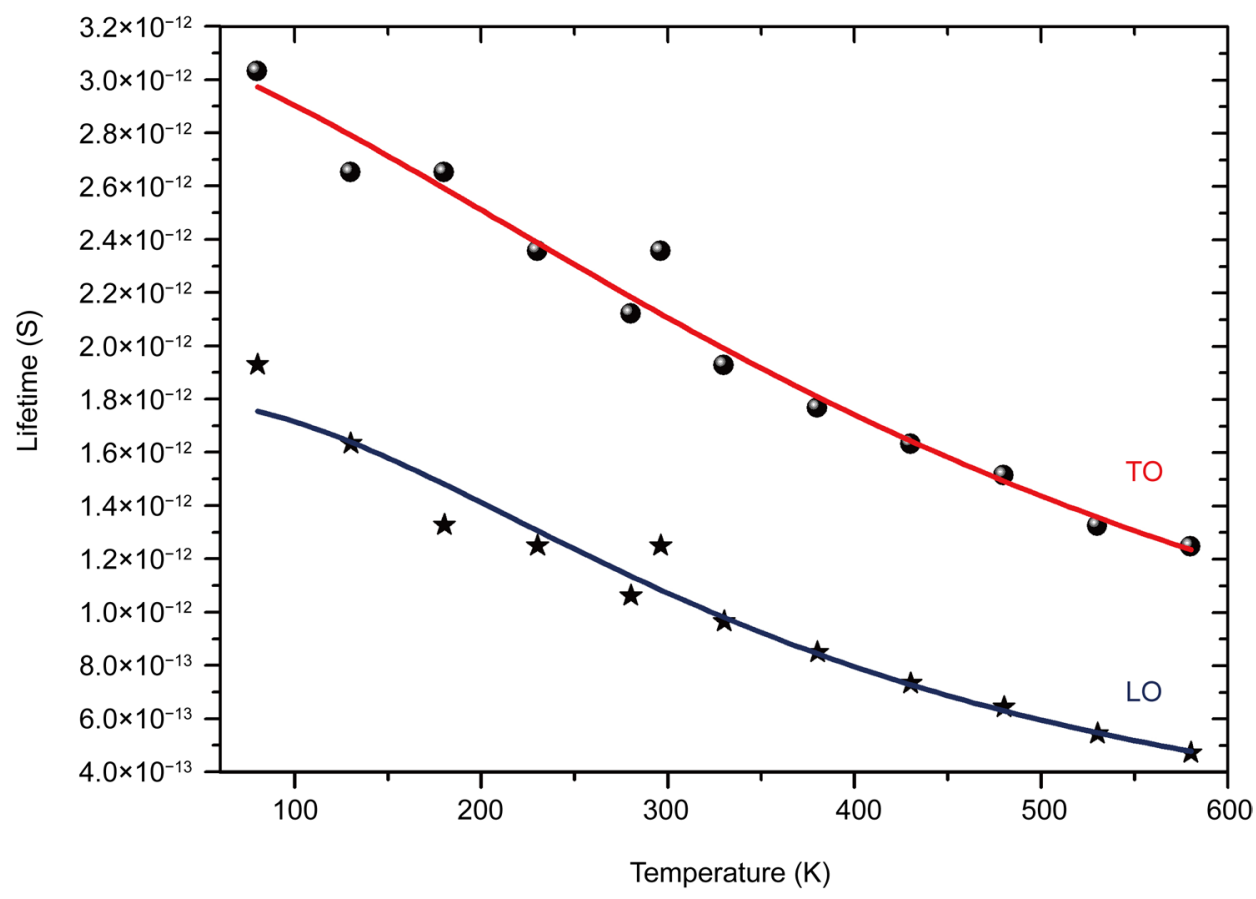

Figure 5. Temperature dependence of the lifetime for TO and LO phonons.

nons will participate in the Raman scattering process at a higher temperature. The multiphonon peak $\mathrm{TA}(X, K)+\mathrm{LA}(X)$ at $290 \mathrm{~cm}^{-1}$ shows the same propertiesas the fundamental phonon modes are sensitive to the change of measurement temperature. For the 2TA $(L)$ phonon mode, there are no appreciable temperature dependent changes observed in the both line center and linewidth, indicating that the 2TA $(L)$ mode does not show the anharmonic effects. However, the intensity of the 2TA $(L)$ mode of the $\mathrm{ZnSe}$ nanowires is also greatly dependent on the temperature, which is shown in Figure 6(a). Because the phonons are bosons, which are subjected to the Bose-Einstein distribution, it results in the increasing of number of phonons with elevating temperature. Thus the increasing phonons enhance the Raman scattering intensity. The intensity of secondorder Raman modes can be described as [44]:

$$
I=K \cdot \mathrm{e}^{-\hbar \omega / k_{B} T} \cdot\left(1-\mathrm{e}^{-\hbar \omega / k_{B} T}\right)^{-2}
$$

where $K$ is constant term, $\hbar$ is reduced Planck constant, $\omega$ is phonon frequency, $k_{B}$ is the Boltzmann constant. Figure 6(b) shows a good agreement between the calculated curve and experiment values by using formula (3).

From the theoretical point of view, the surface mode can be detected by Raman scattering when the size is comparable with the wavelength of the incident laser light [38]. The dispersion relation $\omega_{\mathrm{so}}(q)$ for a surface mode in an infinite long cylindrical nanowire can be written as [45]:

$$
\omega_{\mathrm{SO}}^{2}=\omega_{\mathrm{TO}}^{2}+\frac{\tilde{\omega}_{P}^{2}}{\varepsilon_{\infty}+\varepsilon_{m} f(q r)}
$$




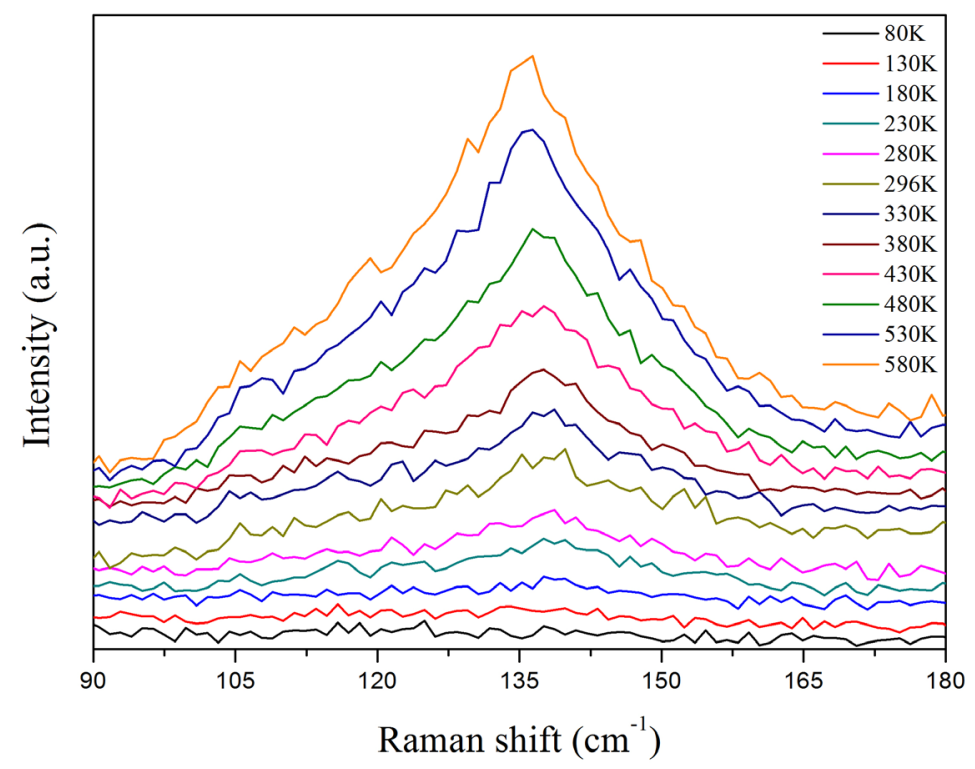

(a)

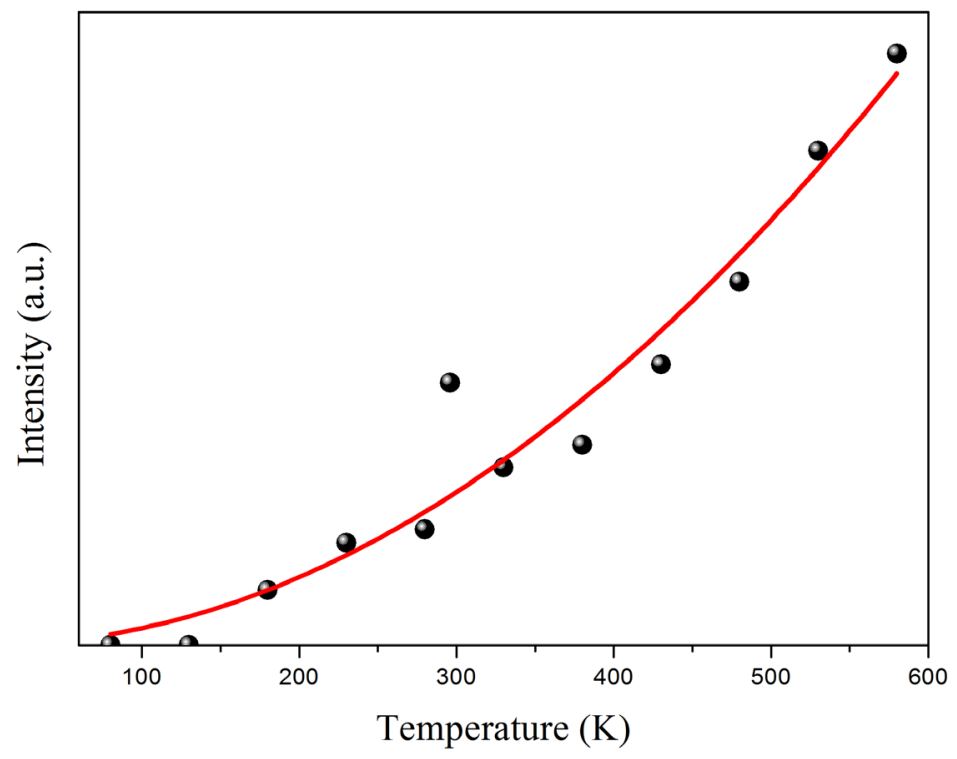

(b)

Figure 6. Temperature-dependent (a) 2TA(L) mode of ZnSe nanowires and (b) its intensity variation with temperature.

where $\tilde{\omega}_{P}$ is the screened ion plasma frequency given by $\omega_{L O}^{2}=\omega_{T O}^{2}+\tilde{\omega}_{P}^{2} / \varepsilon_{\infty}, \quad \varepsilon_{\infty}$ is the high frequency dielectric constant, which is equal to 5.7 [46], $\varepsilon_{m}$ is the dielectric constant of surrounding medium, $r$ is the radius of nanowires. $f(\boldsymbol{q r})$ can be written as:

$$
f(q r)=\frac{I_{0}(q r) K_{1}(q r)}{I_{1}(q r) K_{0}(q r)}
$$

Here $I_{j}(\boldsymbol{q} \boldsymbol{r})$ and $K_{j}(\boldsymbol{q} \boldsymbol{r})$ are the Bessel functions. For the different value of $\boldsymbol{q} \boldsymbol{r}$, the $\omega_{S O}$ can vary from 209 to $249 \mathrm{~cm}^{-1}$. Thus, we tend to assign the weak peak at $243.0 \mathrm{~cm}^{-1}$ got 
at $80 \mathrm{~K}$ to the surface mode [37] [38]. It is found that the energy of surface mode in ZnSe nanowiresis larger than in nanoparticles at the same temperature [38], which may be attributed to the different microtopography of $\mathrm{ZnSe}$. It is noted that the surface modes are observed only under low temperature and will disappear with the temperature increasing, which can be attributed to the anharmonic effects of LO modes.

\section{Conclusion}

The temperature dependent vibrational properties of ZnSe nanowires were investigated by using Raman spectroscopy in $80 \mathrm{~K}-580 \mathrm{~K}$. All the scattering peaks were duly assigned to unitary modes. The asymmetrical broadening of phonon lines are attributed to the anharmonic vibration. Compared with $\mathrm{ZnSe}$ nanoparticles and bulk counterparts, it is found that the phonon lifetimes in ZnSe nanowires are reduced significantly with the dimension reducing, which can be attributed to the larger surface to volume ratios in nanostructures. The surface mode is also observed in $\mathrm{ZnSe}$ nanowires at a low temperature, while some overtones acoustics phonon and multi-phonon are also observed at high temperature. Different phonon behaviors imply a series unusual nature of $\mathrm{ZnSe}$ nanowires compared with bulk counterpart. When implementing these $\mathrm{ZnSe}$ nanowires based devices, many of the physical parameters should be handled carefully. All of these results lay the experimental foundation for fabrication of ZnSe based nanodevices in future.

\section{Acknowledgements}

This work was supported by the National Natural Science Foundation of China (grant number 11174049, 61376017 and 51272159), Natural Science Foundation of Zhejiang province (LY15A040001), Shanghai Talent Development Funding and Graduate dissertation Donghua University Innovation Fund (109-06-0019016).

\section{References}

[1] Jabri, S., Amiri, G., Sallet, V., Souissi, A., Meftah, A., Galtier, P. and Oueslati, M. (2016) Study of the Optical Properties and Structure of ZnSe/ZnO Thin Films Grown by MOCVD with Varying Thicknesses. Physica B, 489, 93-98. https://doi.org/10.1016/j.physb.2016.02.025

[2] Wang, C.R., Wang, J., Li, Q. and Yi, G.C. (2005) ZnSe-Si Bi-Coaxial Nanowire Heterostructures. Advanced Functional Materials, 15, 1471-1477. https://doi.org/10.1002/adfm.200400564

[3] Zhang, X.T., Liu, Z., Leung, Y.P., Li, Q. and Hark, S.K. (2003) Growth and Luminescence of Zinc-Blende-Structured ZnSe Nanowires by Metal-Organic Chemical Vapor Deposition. Applied Physics Letters, 83, 5533-5535. https://doi.org/10.1063/1.1638633

[4] Lohar, G.M., Dhaygude, H.D., Patil, R.A., Ma, Y.R. and Fulari, V.J. (2015) Studies of Properties of $\mathrm{Fe}^{2+}$ Doped Zn Senano-Needles for Photoelectrochemical Cell Application. Journal of Materials Science: Materials in Electronics, 26, 8904-8914. https://doi.org/10.1007/s10854-015-3572-4

[5] Koo, S.M., Fujiwara, A., Han, J.P., Vogel, E.M., Richter, C.A. and Bonevich, J.E. (2004) High Inversion Current in Silicon Nanowire Field Effect Transistors. Nano Letters, 4, 2197 - 
2201. https://doi.org/10.1021/nl0486517

[6] Zhang, X.W., Tang, Z.J., Hu, D., Meng, D. and Jia, S.W. (2016) Nanoscale p-n Junctions Based on p-Type ZnSe Nanowires and Their Optoelectronic Applications. Materials Letters, 168, 121-124. https://doi.org/10.1016/j.matlet.2016.01.044

[7] Garnett, E. and Yang, P.D. (2010) Light Trapping in Silicon Nanowire Solar Cells. Nano Letters, 10, 1082-1087. https://doi.org/10.1021/nl100161z

[8] Hu, Z.D., Duan, X.F., Gao, M., Chen, Q. and Peng, L.M. (2006) ZnSe Nanobelts and Nanowires Synthesized by a Closed Space Vapor Transport Technique. Journal of Physical Chemistry C, 111, 2987-2991. https://doi.org/10.1021/jp067556e

[9] Wang, J.L., Feng, H., Fan, W.L., Chen, K.M. and Yang, Q. (2013) Wet-Chemical Synthesis and Optical Property of ZnSe Nanowires by $\mathrm{Ag}_{2} \mathrm{Se}$-Catalyzed Growth Mechanism. Advances in Materials Physics and Chemistry, 3, 289-294.

https://doi.org/10.4236/ampc.2013.36039

[10] Huang, J., Zhang, H.J., Lucero, A., Cheng, L.X., Santosh, K.C., Wang, J., Hsu, J., Cho, K. and Kim, J. (2016) Organic-Inorganic Hybrid Semiconductor Thin Films Deposited Using Molecular-Atomic Layer Deposition (MALD). Journal of Materials Chemistry C, 4, 23822389. https://doi.org/10.1039/C5TC03714J

[11] Lee, B.C., Kim, K.W., Stroscio, M.A. and Dutta, M. (1998) Optical-Phonon Confinement and Scattering in Wurtzite Heterostructures. Physical Review B, 58, 4860-4865.

[12] Zhang, Y., Han, C., Eerdunchaolu and Sudu (2016) Influence of the Rashba SOI and LO Phonon Effects on the Interaction Energy of the Fröhlich Bipolaron in a Quantum Dot. Journal of the Korean Physical Society, 69, 1301-1309. https://doi.org/10.3938/jkps.69.1301

[13] Wright, A.D., et al. (2016) Electron-Phonon Coupling in Hybrid Lead Halide Perovskites. Nature Communications, 7, Article No. 11755. https://doi.org/10.1038/ncomms11755

[14] Pastor, D., Cuscó, R., Artús, L., Díaz, G.G., Fernández, S. and Calleja, E. (2005) Effect of the Implantation Temperature on Lattice Damage of $\mathrm{Be}^{+}$-Implanted GaN. Semiconductor Science and Technology, 20, 374-377. https://doi.org/10.1088/0268-1242/20/5/009

[15] Zhang, M., et al. (2016) An Experimental and Theoretical Study on Isotopic Surface-Enhanced Raman Spectroscopy for Surface Catalytic Coupling Reaction on Silver Electrodes. Physical Chemistry C, 120, 11956-11965. https://doi.org/10.1021/acs.jpcc.6b02252

[16] Wang, X., et al. (2016) Temperature Dependence of Raman Scattering in $\beta$-(AlGa) $\mathrm{O}_{3}$ Thin Films. AIP Advances, 6, Article ID: 015111. https://doi.org/10.1063/1.4940763

[17] Serrano, J., Manjo'n, F.J., Romero, A.H., Widulle, F., Lauck, R. and Cardona, M. (2003) Dispersive Phonon Linewidths: The $\mathrm{E}_{2}$ Phonons of ZnO. Physical Review Letters, 90, Article ID: 055510. https://doi.org/10.1103/PhysRevLett.90.055510

[18] Bergman, L., et al. (1999) Raman Analysis of Phonon Lifetimes in AlN and GaN of Wurtzite Structure. Physical Review B, 59, 12977-12982. https://doi.org/10.1103/PhysRevB.59.12977

[19] Irmer, G., et al. (2009) Fröhlich Vibrational Modes in Porous ZnSe Studied by Raman Scattering and Fourier Transform Infrared Reflectance. Journal of Physics D: Applied Physics, 42, Article ID: 045405. https://doi.org/10.1088/0022-3727/42/4/045405

[20] An, H.Z., Zhao, Q. and Du, W.M. (2004) Raman Spectra of ZnSe Nanoparticles Synthesized by Thermal Evaporation Method. Chinese Physics, 13, 1753-1757. https://doi.org/10.1088/1009-1963/13/10/032

[21] Yin, S.B., Chen, L. and Hsien, W.F. (1998) Fabrication and Raman Analysis of ZnSe Quantum Dots in Glass Matrix Thin Films by Pulsed Laser Evaporation. Japanese Journal of Ap- 
plied Physics, 37, 4154-4157. https://doi.org/10.1143/JJAP.37.4154

[22] Nesheva, D., Šcepanovic, M.J., Aškrabić, S., Levi, Z., Bineva, I. and Popovic, Z.V. (2009) Raman Scattering from ZnSe Nanolayers. Acta Physica Polonica A, 116, 75-78. https://doi.org/10.12693/APhysPolA.116.75

[23] Wang, H.Y., et al. (2012) Different Temperature Dependence of Excitonic and Defect-Related Photoluminescence Spectra in ZnS Nanobelts and Nanowires. Journal of Physics D: Applied Physics, 45, Article ID: 095301. https://doi.org/10.1088/0022-3727/45/9/095301

[24] Waldecker, L., Bertoni, R., Vorberger, J. and Ernstorfer, R.(2016) Electron-Phonon Coupling and Energy Flow in a Simple Metal beyond the Two-Temperature Approximation. Physical Review X, 6, Article ID: 021003. https://doi.org/10.1103/PhysRevX.6.021003

[25] Xu, J., et al. (2014) ZnSe-Based Longitudinal Twinning Nanowires. Advanced Engineering Materials, 16, 459-465. https://doi.org/10.1002/adem.201300405

[26] Wang, F.F., et al. (2007) Structure and Stimulated Emission of ZnSe Nanoribbons Grown by Thermal Evaporation. Nanotechnology, 18, Article ID: 305705.

[27] Deneuville, A., Tanner, D. and Holloway, P.H. (1991) Optical Constants of ZnSe in the Far infrared. Physical Review B, 43, 6544-6550. https://doi.org/10.1103/PhysRevB.43.6544

[28] Irwin, J.C. and La Combe, J. (1970) Second-Order Raman Spectrum of ZnSe. Canadian Journal of Physics, 48, 2499-2506. https://doi.org/10.1139/p70-314

[29] Bouarissa, N., Siddiqui, S.A., Hajry, A., Saib, S. and Boucenna, M. (2014) Elastic Properties and Lattice Vibration Modes in $\mathrm{ZnTe}_{1-\mathrm{x}} \mathrm{O}_{\mathrm{x}}$. Computational Materials Science, 83, 22-26. https://doi.org/10.1016/j.commatsci.2013.10.028

[30] Balaguru, R.J.B. and Jeyaprakash, B.G. (2015) Lattice Vibrations, Phonons, Specific Heat Capacity, Thermal Conductivity. http://www.nptel.ac.in/courses/115106076/Module\%209/Module\%209.pdf

[31] Caldwell, J.D., et al. (2015) Low-Loss, Infrared and Terahertz Nanophotonics Using Surface Phonon Polaritons. Nanophotonics, 4, 44-68. https://doi.org/10.1515/nanoph-2014-0003

[32] Farley, K.E. (2016) Electron-Phonon Coupling and Structural Phase Transitions in Early Transition Metal Oxides and Chalcogenides. Ph.D. Thesis, State University of New York at Buffalo, Buffalo.

http://xueshu.baidu.com/s?wd=paperuri\%3A\%28e606871442d8428028d3ea2ff31b6089\%29 \&filter=sc_long_sign\&tn=SE_xueshusource_2kduw22v\&sc_vurl=http\%3A\%2F\%2Fadsabs. harvard.edu\%2Fabs\%2F2016PhDT.........31F\&ie=utf-8\&sc_us=3178911112751214064

[33] Wang, C.R., Ip, K.M., Hark, S.K. and Li, Q. (2005) Structure Control of CdS Nanobelts and Their Luminescence Properties. Journal of Applied Physics, 97, Article ID: 054303.

[34] Lu, G.W., et al. (2005) Temperature Dependence of Raman Scattering of ZnSe Nanoparticle Grown through Vapor Phase. Journal of Crystal Growth, 274, 530-535. https://doi.org/10.1016/j.jcrysgro.2004.10.062

[35] Yu, Y., et al. (2009) Ab Initio Study of Structural, Dielectric, and Dynamical Properties of Zinc-Blende $\mathrm{ZnX}(\mathrm{X}=\mathrm{O}, \mathrm{S}, \mathrm{Se}, \mathrm{Te})$. Journal of Alloys and Compounds, 471, 492-497. https://doi.org/10.1016/j.jallcom.2008.04.039

[36] Kushwaha, A.K. (2010) Phonon Spectrum and Thermal Properties of Semiconducting Compounds ZnS and ZnSe. Physica B: Condensed Matter, 405, 1638-1642. https://doi.org/10.1016/j.physb.2009.12.059

[37] Shan, C.X., Liu, Z., Zhang, X.T., Wong, C.C. and Hark, S.K. (2006) Wurtzite ZnSe Nanowires: Growth, Photoluminescence, and Single-Wire Raman Properties. Nanotechnology, 17, 5561-5564. https://doi.org/10.1088/0957-4484/17/22/006 
[38] Fu, X.G., An, H.Z. and Du, W.M. (2005) Temperature-Dependent Raman Scattering Studies in ZnSe Nanoparticles. Materials Letters, 59, 1484-1490. https://doi.org/10.1016/j.matlet.2005.01.002

[39] Verma, P., Abbi, S.C. and Jain, K.P. (1995) Raman-Scattering Probe of Anharmonic Effects in GaAs. Physical Review B, 51, 16660-16667. https://doi.org/10.1103/PhysRevB.51.16660

[40] Chen, Y.Q., Peng, B. and Wang, B. (2007) Raman Spectra and Temperature-Dependent Raman Scattering of Silicon Nanowires. Journal of Physical Chemistry C, 111, 5855-5858. https://doi.org/10.1021/jp0685028

[41] Pandey, S.D., Singh, J., Samanta, K., Sharma, N.D. and Bandyopadhyay, A.K. (2015) Temperature Dependent Variations of Phonon Interactions in Nanocrystalline Cerium Oxide. Journal of Nanomaterials, 2015, Article ID: 492967. https://doi.org/10.1155/2015/492967

[42] Anand, S., Verma, P., Jain, K.P. and Abbi, S.C. (1996) Temperature Dependence of Optical Phonon Lifetimes in ZnSe. Physica B: Condensed Matter, 226, 331-337. https://doi.org/10.1016/0921-4526(96)84974-X

[43] Su, Z., et al. (2006) Temperature-Dependent Raman Scattering of Silicon Nanowires. The Journal of Physical Chemistry B, 110, 1229-1234. https://doi.org/10.1021/jp055869o

[44] Chang, W., Zhu, K.R. and Ma, Y.Q. (2011) Temperature-Dependent Raman Spectrum of ZnO Nanocrystals Synthesized by Technique of Solid-Phase Reaction. Journal of Hefei University of Technology, 34, 997-1000.

[45] Xiong, Q.H., et al. (2007) Surface Optic Phonons in Cylindrical and Rectangular CrossSectional Semiconducting Nanowires. Nanoscience and Technology, 121-123, 955-966.

[46] Bartolo, B.D. and Chen, X.S. (2001) Advances in Energy Transfer Processes. In: Wegener M., Ed., Non-Markovian Scattering Processes in Semiconductors, World Scientific Publishing, Singapore, 222.

http://www.worldscientific.com/doi/pdf/10.1142/9789812810960_0006

Submit or recommend next manuscript to SCIRP and we will provide best service for you:

Accepting pre-submission inquiries through Email, Facebook, LinkedIn, Twitter, etc.

A wide selection of journals (inclusive of 9 subjects, more than 200 journals)

Providing 24-hour high-quality service

User-friendly online submission system

Fair and swift peer-review system

Efficient typesetting and proofreading procedure

Display of the result of downloads and visits, as well as the number of cited articles

Maximum dissemination of your research work

Submit your manuscript at: http://papersubmission.scirp.org/

Or contact ampc@scirp.org 